\title{
E1 humanismo de Illich, evaluación de tecnologías para una sociedad convivencial
}

Matías Aimino*

Resumen: La crítica de Illich a las sociedades industriales contemporáneas involucra, entre otras cosas, una nueva concepción de la elección o evaluación de tecnologías basada en la noción filosófica de "convivencialidad". El propósito de este trabajo es reconstuir esta concepción, cuyos rasgos más salientes son el pluralismo radical, el constructivismo tecnológico y el bumanismo, esto es, la idea de que la vara más adecuada para medir los efectos reales o potenciales del desarrollo de tecnologías es la preservación de la autonomía humana.

Palabras clave: Racionalidad, evaluación de tecnologías, pluralismo, límites al crecimiento, convivencialidad.

Abstract: Illich's criticism of contemporary industrial societies involves, among other things, a new conception of technology assessment based on the philosophical notion of "conviviality". The purpose of this paper is to reconstruct such conception, whose most salient features are radical pluralism, technologic constructivism and bumanism, that is, the idea that the best yardstick to measure actual or potential effects of technology development is the preservation of human autonomy.

Keywords: Rationality, technology assessment, pluralism, limits to growth, conviviality.

\section{Introducción}

Iván Illich es considerado, acertadamente, como el más lúcido de los críticos de las sociedades industriales contemporáneas, como el responsable de que éstas hayan perdido definitivamente cualquierjustificación teórica.

* Doctor en filosofía, arquitecto. Docente-investigador de la Universidad Nacional del Litoral. Áreas: filosofía de la ciencia, filosofía de la tecnología, arquitectura. Autor de varios artículos en las áreas mencionadas y en relación con el presente trabajo de: "Racionalidad y evaluación de tecnologías. El caso de las arquitecturas de tierra cruda”, en Lawler, D., Parente, D., Blanco, J., ed., Mundos técnicos. Aspectos ontológicos, normativos y epistemológicos de la artificialidad, Buenos Aires, Universidad Abierta Interamericana, 2012. Dirección electrónica: aiminomatias@gmail.com 
Durante los años de existencia del CIDOC ${ }^{1}$ en Cuernavaca (1966-1976),Illich publica una serie de libros, ${ }^{2} a$ los que en conjunto designa como «mis panfletos», en los que denuncia, entre otras cosas, la contraproductividad intrínseca detodas las instituciones creadas por las sociedades industrialescon el propósito de producir bienes y servicios. Mientraslos miembros del Club de Roma, advertidos de que la naturaleza ya no podría tolerar las crecientes agresiones de la industria, ${ }^{3}$ proponen por esos años un proceso de desmaterialización de la economía (esto es, un desacoplamiento entre el crecimiento económico y su base material) así como una progresiva reorientación hacia la producción de servicios, Illich demuestra la completa implausibilidad de la propuesta, señalando "que, pasados ciertos umbrales, la producción de servicios sería más destructora de la cultura que lo que la producción de bienes materiales lo es de la naturaleza."4

La única posibilidad de contener los efectos realmente destructores de la industria sobre la naturaleza, la sociedad y la cultura consiste, para Illich, en limitar políticamente la producción industrial de bienes y servicios o, en otras palabras, en imponer límites al crecimiento de la economía industrial.El hecho de que estos límites solamente puedan establecerse mediante un proceso político supone que las personas y las comunidades deben tener a disposición los instrumentos analíticos adecuados para identificar los límites admisibles por debajo de los cuales deben contenerse la magnitud y la intensidad de uso de las instituciones productoras de bienes y servicios. Con el objetivo de proporcionar algunos de estos instrumentos analíticos, Illich introduce una serie de nociones tales como herramientas, umbrales críticos de desarrollo, productividad y contraproductividad, imperativo tecnologico, norma de lo suficiente,

${ }^{1}$ Centro Intercultural de Documentación, fundado por Illichy otros colaboradores tras la disolución del CIF, Centro Intercultural de Formación.

${ }^{2}$ Esos libros son:Alternativas (1970/1974), La sociedad desescolarizada (1970), Energía y equidad (1973), La convivencialidad(1973) y Némesis médica(1975). Se han compilado en Illich, Iván, Obras reunidas I, revisión de Valentina Borremans y Javier Sicilia, México, FCE, 2006.

${ }^{3} \mathrm{La}$ advertencia proviene del primer informe Meadows (1972). Cf. Meadows, Donella, Limits to growth. A report for the Club of Rome's project on the predicament of mankind, New American Library, 1977.

${ }^{4}$ Robert, Jean y Borremans, Valentina, "Prefacio" a Illich, op. cit. (2006), p. 18. 
autonomía humana, convivencialidad. ${ }^{5}$ Estas nociones no solamente son nucleares de la crítica de Illich a las sociedades industriales contemporáneas, sino también de lo que podría designarse más precisamente como su propia filosofía de la tecnología. En efecto, fuertemente imbricado con su pensamiento crítico, hay un contenido propositivo ciertamente potente en la filosofía de Illich que puede considerarse como una nueva concepción de la elección o evaluación de tecnologías. Los rasgos más salientes de esta concepción, cuya reconstrucción constituye el propósito de este trabajo, son el pluralismo radical (la idea de que la apertura del espacio político de la elección o evaluación de tecnologías solamente se logra al considerar alternativas tecnológicas radicalmente diferentes), el constructivismo tecnológico(la idea de que la consideración de alternativas radicalmente diferentes promueve un proceso de interacciones que es eminentemente constructivo y que conduce al desarrollo de tecnologías en algún sentido mejores o superadoras) y por último el bumanismo(la idea de que lavara más adecuada para medirlos efectos reales o potenciales deldesarrollo de tecnologíases la preservación de la autonomía humana). En este punto, lo importante para Illich es saber si, en definitiva, las tecnologías "ayudan u obstaculizan la capacidad del hombre para vivir en la plenitud y la alegría."6

\section{Herramientas y umbrales críticos}

La filosofía de la tecnología de Illich puede caracterizarse como una filosofía de las herramientas, donde el término «herramienta» se usa en un sentido amplio, con el propósito de incluir no solamente los artefactos construidos por el hombre, sino también las instituciones humanas dedicadas a la producción de bienes y servicios (como las fábricas y las centrales eléctricas, los sistemas de transporte, los hospitales y los establecimientos educativos). De acuerdo con Illich, "la categoría de la herramienta engloba todos los instrumentos razonados de la acción humana, la máquina y su modo de

${ }^{5} \mathrm{El}$ término «convivencialidad», en francés convivialité, proviene de la Physiologie du goût (1825) de Jean Anthelme Brillat-Savarin.

${ }^{6}$ Fromm, Erich, “Introducción” a Alternativas, en Illich, op. cit. (2006), p. 48. 
empleo, el código y su operador, el pan y el circo. (...) Todo objeto tomado como medio para un fin se convierte en herramienta." 7

El hombre necesita de las herramientas porque éstas le proporcionan bienes y servicios. Las herramientas, aunque varían sustancialmente de una cultura a la otra y de un período histórico al otro, pueden ordenarse en una serie continua cuyos extremos son la herramienta convivencial o manejable y la herramienta dominante o manipuladora. En principio, la diferencia entre estos dos tipos de herramientas no reside en el nivel de complejidad tecnológica que hayan alcanzado. Las herramientas convivenciales permiten transformar la energía metabólica humana con el propósito de realizar una tarea más eficientemente; así, expanden el radio de acción personal, pero sin crear ninguna dependencia, sin degradar la autonomía humana. Las herramientas convivenciales tienen un propósito que no consiste en su propio uso repetido y, por eso mismo, son autolimitativas (una vez logrado el propósito, cesa el uso de las herramientas y éstas simplemente quedan a disposición). Cuando adoptan una forma institucional, las herramientas convivenciales son redes sociales que facilitan la comunicación y la cooperación de sus miembros. Las herramientas dominantes, por el contrario, demandan un constante incremento de la energía externa (no metabólica), alientan la concentración económica y la centralización política, crean dependencia, y amenazan gravemente la autonomía humana. El principal propósito de las herramientas dominantes es su propio uso repetido, su propia reproducción. Para lograrlo, las herramientas dominantes suscitan sobre los usuarios una continua serie de manipulaciones, los hacen víctimas de la propaganda, del adoctrinamiento, de la agresión. Cuando adoptan una forma institucional, las herramientas dominantes son procesos de producción complejos y dispendiosos en los que una gran parte de los gastos se destinan a convencer alos consumidores de que no pueden vivir sin los bienes y servicios proporcionados por la institución. En las sociedades basadas en el modo de producción industrial, las herramientas convivenciales paulatinamente han cedido el paso a las herramientas dominantes, y esto ha sido el resultado de superar o trasponer dos umbrales críticos. El primer umbral crítico se traspone cuando la producción de un determinado bien o servicio

${ }^{7}$ Illich, op. cit. (2006), p. 396. 
se generaliza, y éste se transforma en un producto de consumo social. El segundo umbral crítico se traspone cuando la producción generalizada de un determinado bien o servicio deja de contribuir al logro del mismo propósito para el que dicho bien o servicio fue producido. ${ }^{8} \mathrm{El}$ primer umbral crítico es el umbral de la productividad, mientras que el segundo es el de la contraproductividad. Illich ilustra la superación de estos umbrales por medio de cuatro ejemplos de tecnologías sociales que siguen un desarrollo notablemente análogo.

Salud. Las culturas no industriales afrontan el dolor, la enfermedad y la muerte interpretándolos, y derivan su función sanitaria precisamente de su capacidad de equipar a las personas "para hacerles el dolor tolerable, la enfermedad o la invalidez comprensible y la sombra de la muerte significativa". ${ }^{\circ}$ En cambio, las sociedades industriales tratan de afrontar estos problemas suprimiéndolos. Convierten el dolor, la enfermedad y la muerte en problemas técnicos que deben ser resueltos, y crean un entramado de instituciones sanitarias que ponen en manos de médicos profesionales e imponen a toda la población bajo la forma de un servicio social. A inicios del siglo XX, la medicina institucional se universaliza y supera el umbral de la productividad al desarrollar y difundir una serie de innovaciones científicotecnológicas que significan mayor bienestar para un mayor número de personas. Pero, a mediados del siglo $\mathrm{XX}$, la medicina institucional se aproxima al umbral de la contraproductividad cuando se hacen frecuentes las enfermedades iatrogénicas, esto es, las enfermedades que tienen por agentes patógenos a los medicamentos, los médicos o los hospitales. Una vez traspuesto el segundo umbral, la medicina institucional produce mayor sufrimiento para un mayor número de personas, su función sanitaria declina, su eficacia se convierte en mera ilusión. ${ }^{10}$ Lo que queda por hacer no es más

${ }^{8}$ Desde la perspectiva de Illich, la racionalidad humana involucra una dimensión teleológica y una dimensión instrumentalque deben pensarse conjuntamente y que no es prudente escindir. Cualquier deliberación sobre fines que no considera la escala bumana de los medios puede conducir a resultados irracionales y hacer que los medios se vuelvan fines en sí mismos. 'Illich, op. cit. (2006), p. 638.

${ }^{10}$ Las consideraciones de Illich son realmente alarmantes cuando se las contrasta con los datos estadísticos. Cfr. por ejemplo el informe del U.S. Institute of Medicine, To 
que enmascararsu contraproductividad desarrollando más medicina institucional para afrontar los males que ella misma ha engendrado.

Educación. En la educación ocurre algo análogo a lo que ocurre en la salud. El aprendizaje es una actividad que los seres humanos desempeñan de manera espontánea, a menudo cuando practican actividades que reciben otros nombres, como «trabajo» o «juego». Sin embargo, el desarrollo de las instituciones educativas no se basa en esta comprensión del aprendizaje, sino en la ideade que el aprendizaje es el producto de la instrucción, de la enseñanz̧a programada mediante un currículo jerarquizado. Durante el siglo XIX, las sociedades industrialesimponen la escolaridad universal y obligatoria, y así las instituciones educativas franquean el primer umbral de desarrollo, que es el umbral del monopolioy de la productividad. Sin embargo, la escolaridad obligatoria es económicamente impracticable si pretende asimismo ser universal, puesto que ningún estado contemporáneo puede satisfacer la demanda de un sistema escolar cada vez más amplio, con grados más elevados y realmente accesible a todos. La imposibilidad de satisfacer esta demanda suscita entonces una situación contraria al mismo propósito de la educación universal. El sistema escolar restringe progresivamente el acceso a los grados superiores yde este modo legitima la estratificación de la sociedad según el nivel de escolaridad alcanzado. Así, las instituciones educativas superan el segundo umbral de la contraproductividad. La educación universal, que constituye un propósito ciertamente deseable, no puede sin embargo alcanzarse cuando la escolaridad obligatoria monopoliza su distribución. Por eso, Illich defiende la propuesta(neoilustrada) de desescolarizar la sociedad y de restituir a todas las personas la responsabilidad de su propio aprendizaje.

Transporte. Los seres humanos, así como tienen la capacidad de curarse y deaprender por sí mismos, también tienen la capacidad de desplazarse por sí mismos yrecorrer grandes distancias con movimientos basados en la fuerza de sus músculos, en su propia energía metabólica. Illich denomina "tránsito" a este modo de circulación o desplazamiento de personas y lo opone a otro modo de circulación, el "transporte", que se caracteriza por trasladar a las personas en vehículos motorizados. El transporte es el modo de circulación

err is human. Building a safer health system, Washington, The National Academies Press, 2000. 
que han desarrollado prevalentemente las sociedades industriales al difundir la producción y el consumo de vehículos motorizados y al promover la construcción de una vasta red de calles, avenidas, rutas y autopistas que son subsidiarias de este tipo de circulación. Cuando el transporte monopoliza la circulación supera el primer umbral de la productividad y se dispone a aproximarse al segundo umbral de la contraproductividad. El transporte se torna contraproductivo cuando le cuesta a la sociedad más tiempo del que le ahorra. ${ }^{11}$ Los habitantes de los grandes conglomerados urbanos contemporáneos como Nueva York o Tokio, pero también como Córdoba o Santa Fe, invierten gran parte de su tiempo en desarrollar y mantener un sistema de transporte que los traslada diariamente a una velocidad inferior a la que alcanzarían yendo en bicicleta,pero que les hace asimismo impracticable desplazarse por sí mismos en bicicleta.

Vivienda. En todos los tiempos los seres humanos han aprovechado los materiales de su entorno inmediato para construir por sí mismos sus moradas. Sin embargo, las sociedades industriales han logrado, con la implementación de códigos de edificación urbana, expropiar a los hombres esta posibilidad de autoconstrucción. Los códigos establecen normas técnicas de edificación y condiciones mínimas de habitabilidad basándose enel modelo de la vivienda profesionalmente construida. Al priorizar este modelo, los códigos desalientan la autoconstruccióny originan una creciente demanda de viviendas, porque ningún trabajador que en su tiempo libre construye por sí mismo su morada puede satisfacer las normas impuestas por el código. Para responder a esta demanda, los capitales públicos y privados abren el juego de los créditos hipotecarios e impulsan la industria de la construcción, que así consigue el monopolio de la producción de viviendas y transpone elumbral de la productividad. Cuando la producción de un bien o servicio supera este primer umbral, sustituye progresivamente su propósito originario por un nuevo propósito que consiste en el permanente incremento de la producción. La industria de la construcción supera el segundo umbral de desarrollo, el de la contraproductividad, cuando produce una sobreabundancia de viviendas y al mismo tiempo un alza creciente de los precios de los bienes inmuebles, una notable concentración de los títulos de

${ }^{11}$ Cf. también Gorz,André, Ecológica, Madrid, Clave Intelectual, 2012, pp. 59-72. 
propiedad y un endeudamiento progresivo de gran parte de la población, obligada a pagar una deuda vitalicia en concepto de alquileres o de hipotecas. La paradoja mayor de todo este proceso es que aumenta notablemente el número de viviendas vacantes, pero continúa sin ser satisfecha la demanda habitacional real.

Los cuatro ejemplos de tecnologías sociales tratados precedentemente revelan la existencia de dos modos de resolver el ciclo denecesidad y satisfacción, uno basado en la producción orgánica, autónoma, personal y comunitaria, y otro basado en la producción mecánica, heterónoma, industrial. Estos dos modos de producción pueden complementarse sin entrar en conflicto cuando el desarrollo de la industria se mantiene por debajo del primer umbral crítico y de este modo se evita el monopolio sobre la producción de un determinado bien o servicio. Según Illich,

la producción autónoma puede complementarse con productos industriales. Puede hacerse más eficaz y más descentralizada utilizando herramientas fabricadas industrialmente como bicicletas, libros $\mathrm{O}$ antibióticos. Pero también puede ser obstaculizada, devaluada y bloqueada por un reordenamiento de la sociedad totalmente a favor de la industria. ${ }^{12}$

Cuando se da prioridad al modo de producción industrial, las herramientas superan su límite admisible de desarrollo, se vuelven contra los propósitos para los que fueron pensadas y se convierten en una seria amenaza para la sobrevivencia humana.

\section{El imperativo tecnológico y la transgresión de la norma de lo suficiente}

La aceleración sin precedentes que, desde hace al menos un siglo, signa el desarrollo tecnológico en las sociedades industriales contemporáneas tiene su base, de acuerdo con Illich, en la aceptación del imperativo tecnológico y en la transgresión de la norma de lo suficiente. El imperativo tecnológicoconstituye uno de los principios normativos más importantes del

12 Illich, Iván, Némesis médica. La expropiación de la salud, Barcelona, Barral, 1975, p. 80 (el fragmento citado ha sido omitido en la edición de 2006). 
modo de producción industrial, y es independiente del tipo de régimen de propiedad (capitalista o socialista) al que estén sometidos los medios de producción. Este imperativo imponea las sociedades contemporáneas un progreso indefinido en la calidad y cantidad de la producción industrial de bienes y servicios, un progreso que debe lograrse a cualquier costo natural o humano. Al respecto,Illich y Borremans convienen que la expresión "imperativo tecnológico" designa precisamente "la idea de que si alguna hazaña técnica es posible en cualquier parte del mundo, hay que realizarla y ponerla al servicio de algunos hombres, sin importar en lo absoluto el precio que los demás miembros de esa sociedad hayan de pagar por ella."13 El imperativo tecnológico justifica de este modo la concentración económica y la centralización política porque ningún progreso tecnológico, en el sentido antes apuntado, podría alcanzarse sin éstas.

La aceptación del imperativo tecnológico conduce a la transgresión de la norma de lo suficiente, ${ }^{14}$ que marca los límites naturales a los que se encuentra sometido el ciclo de necesidad y satisfacción. En las culturas no industriales, estos límites no pueden transgredirse porque son determinados por un equilibrado ajuste entre lo que los hombres necesitan y lo que tiene para ofrecerles el entorno natural y social. Esas culturas expresan sus necesidades de manera diferente e imprimen un sello diferente a la satisfacción de tales necesidades, pero lo hacen sin transgredir la norma de lo suficiente, sin franquear los límites naturales. En cambio, las sociedades industrialesconstantemente tratan de transgredir esta norma mediante el poder que les confieren la ciencia y la tecnología. Los términos "progreso", "crecimiento" y "desarrollo" son los nombres con los que a menudo se designa la transgresión de los límites naturales impuestos por la norma de lo suficiente. Esta transgresión modifica radicalmente el ciclo de necesidad y satisfacción, porque altera el significado mismo que se atribuye a las necesidades. Como se ha indicado antes, el hombre ya no necesitadesplazarse, sino que necesita "transporte"; no necesita aprender, sino que necesita "educación"; no necesita protegerse de las inclemencias del tiempo o hacerse

${ }^{13}$ Illich, Iván yBorremans, Valentina, "La necesidad de un techo común (el control social de la tecnología)", en Illich,op. cit. (2006),p. 762.

14 Esta norma tiene sus antepasados ilustres en la Utopía deMoro y en El capital de Marx (cf. tomo III, cap. XLVIII, acápite III). 
un lugar donde vivir, sino que necesita "vivienda". Así, en las sociedades industriales, las necesidades se convierten en la condición que hace a los seres humanos dependientesde bienes y servicios. Según Illich, la humanidad "ha llegado a ser definida por las necesidades comunes a todos sus miembros. (...)Como resultado, las necesidades han devenido el fundamento universal de las certezas sociales." 15

La universalización de las necesidades parece introducir una nueva categoría antropológica, porque supone el nacimiento de un nuevo tipo de hombre al que Illich denomina homo miserabilis, el hombre necesitado, el hombre que se reconoce a sí mismo en la permanente demanda de bienes y servicios. Pero las necesidades no solamente son universalizadas, sino también operacionalizadas. En primer lugar, se ordenan en una jerarquía a la que se atribuye un presunto carácter objetivo y, por lo tanto, también normativo. En segundo lugar, se expresan mediante una serie de indicadores cuantitativos que las hacen, en definitiva, económicamente medibles. Los índices de necesidades básicas insatisfechas son la muestra más cabal de esta operacionalización de las necesidades y, al decir de Illich, "el legado más insidioso que deja el desarrollo"16, puesto que la humanidad puede dividirse ahora entre los que están por encima y los que están por debajo de un nivel medible de NBI. De este modo, las necesidades universalizadas y operacionalizadas legitiman el predominio de la ciencia y la tecnología,así como la administración tecnocrática de las sociedades contemporáneas. Las necesidades proporcionan una justificación filantrópica a la aplicación de la ciencia y la tecnología para el control social del homo miserabilis -el nuevo hombre necesitado- $y$, como consecuencia de esto, para el avasallamiento de las culturas no industriales. ${ }^{17}$

La aceptación del imperativo tecnológico y el ideal del desarrollo ilimitado, la transgresión de la norma de lo suficiente y la universalización de las necesidades, plantean inevitablemente la cuestión de la razonabilidad de todo esto. ¿Es razonable promover una demanda permanente y siempre

${ }^{15}$ Illich, Iván, "Necesidades", en Sachs, Wolfgang(ed.), Diccionario del desarrollo, Lima, PRATEC, 1996, p. 159.

${ }^{16}$ Ibidem, p. 157.

${ }^{17}$ Ibidem, p. 169 y ss. 
insatisfecha de bienes y servicios? ¿Es razonable aspirar a un desarrollo ilimitado en un planeta con recursos limitados? La respuesta de Illich es abiertamente negativa, porque las instituciones pensadas para satisfacer las necesidades humanas "crean a mayor velocidad necesidades que satisfacciones $y$, en el proceso de tratar de satisfacer las necesidades que engendran, consumen la tierra. Esto vale para la agricultura y la manufactura, y no menos para la medicina y la educación." 18

Por eso mismo, los ideales de progreso constante, de desarrollo ilimitado, de crecimiento indefinido -incluso aunque se los conciba y se los defienda desde la perspectiva de la sostenibilidad - no hacen más que contribuir al deterioro de losdiversos equilibrios ${ }^{19}$ que hacen posible la existencia humana y, en consecuencia,al agravamiento delas así denominadas "crisis contemporáneas".

El equilibrio de la vida es el equilibrio entre el hombre y la biosfera. Dondequiera que el modo de producción industrial se impone y pone a funcionar sus herramientas dominantes, este equilibrio se destruye. Las consecuencias son el agotamiento de los recursos naturales, la extinción de las especies y la reducción de la biodiversidad, así como la contaminación del entorno natural debida a la polución, la radiación, la emisión de desechos tóxicos, entre otros.

El equilibrio de la energía es el equilibrio entre la energía metabólica y la energía externa (no metabólica) requeridas para afrontar el ciclo de necesidad y satisfacción. En las sociedades industriales este equilibrio se ha destruido porque la satisfacción de las necesidades depende principalmente de la energía externa (no metabólica), que ha superadoen cientos de veces a la energía metabólica global. Esta concentración energética sin precedentes constituye,en varios aspectos, una grave pérdida de la autonomía humana.

El equilibrio del saber es el equilibrio entre el saber adquirido espontáneamente y el saber recibido mediante la educación disciplinada. Según Illich, el saber global de una sociedad prospera si ambos modos de saber se desarrollan al mismo tiempo, porque entonces "el rigor y la libertad

18 Illich, op. cit. (2006), p. 292.

${ }^{19}$ Ibidem, p. 417 y ss. 
se conjugan armoniosamente." 20 Las sociedades industriales han destruido este equilibrio al hacer que la educación disciplinada sea un medio de adoctrinamiento masivo y estandarizado, cuyo mayor propósito es insertar a las personas en el sistema productivo y convertirlas en usuarios y consumidores de los bienes y servicios producidos por ese mismo sistema.

El equilibrio del poder consiste en la distribución equitativade la posibilidad de intervenir activamente en las decisiones. Las sociedades industriales han destruido este equilibrio al concentrar el poder de decisión en manos de una élite intelectual y al despojar a la mayoría de las personas de la posibilidad de hacer valer su palabra. La tecnocraciapropia de las sociedades industriales contemporáneas se basa, según Illich, en el siguiente razonamiento: el poder de decisión debe reservarse a las personas más productivas y la productividad de las personas puede medirse por la inversión educativa de que han sido objeto.

El equilibrio del cambio consiste en una transición prudente, en una relación significativa entre el pasado y el futuro, entre la tradición y la utopía. Para Illich, la propia estructura de las herramientas es la que impone la dirección y el ritmo del cambio. El modo de producción autónoma deja que las personas y las comunidades conserven su cultura y sus formas de vida, y al mismo tiempo permite que las modifiquen espontáneamente. El modo de producción industrial, por el contrario, destruye el equilibrio del cambio al instalar un proceso de innovación permanente y de obsolescencia programada, con el que busca acelerar del ciclo de producción y consumo.

El equilibrio de la acción es el equilibrio entre los fines perseguidos y las acciones necesarias para alcanzarlos, es la justa proporción entre los medios y los fines. Las herramientas dominantes de las sociedades industriales contemporáneas, al volverse contra los propósitos para los que fueron pensadas, destruyen este equilibrio y provocan en los usuarios y consumidores un sentimiento de creciente insatisfacción. La contraproductividad hace que, en definitiva, el precio que los hombres deben pagar por los bienes y servicios industrialmente producidos sea demasiado alto.

${ }^{20}$ Ibidem, p. 429. 
La destrucción de estos equilibrios queda representada en una proliferación de crisis contemporáneas (como la crisis ecológica, la crisis energética, la crisis institucional, etc.) que la agenda científico-tecnológica actual inscribe entre sus preocupaciones centrales, pero que sin embargo pretende resolver con mayor investigación y desarrollo, con tecnologías más eficientes y racionales. Illich está en desacuerdo con esta primera vía del desarrollo sostenible, porque considera que en lugar de resolver las crisis mencionadas tiende inevitablemente a agudizarlas. Según Illich, lograr un modo de producción que sea "a la vez hiperindustrial y ecológicamente realizable es acelerar la degradación de los otros componentes del equilibrio multidimensional de la vida." ${ }^{21}$

Illich propone, en cambio, una segunda vía para restablecer los equilibrios que hacen posible la existencia humana, y ésta es la vía de los límites al crecimiento. Esta vía sugiere que, si se imponen límites el desarrollo de las herramientas dominantes propias del modo de producción industrial, es posible avanzar hacia una sociedad convivencial y posindustrial, hacia una sociedad en la que el crecimiento industrial esté balanceado y mantenido bajo control por una diversidad de modos de producción autónoma. Una sociedad convivencial y posindustrial es aquélla en que los hombres pueden controlar las herramientas y en que las herramientas son eficientes, pero no degradan la autonomía humana, sino que permanecen al servicio de las personas y las comunidades. Esto no significa ni una vuelta al pasado ni una restauración utópica del mito del buen salvaje; en particular, no significa que una sociedad convivencial deba proscribir toda herramienta poderosa y toda producción centralizada. Éstas tienen que admitirse, pero también deben mantenerse por debajo de ciertos límites, por debajo del primer umbral crítico del monopolio y la productividad industrial.

\section{Hacia una sociedad convivencial y posindustrial}

${ }^{21}$ Ibidem, pp. 474-475. 
La posibilidad de avanzar por el camino delos límites alcrecimiento hacia una sociedad convivencial y posindustrial está íntimamente ligada, por razones obvias, a una serie de decisiones que deben tomarse en torno a las tecnologías. Al respecto, aunque Illich no haconcebido un modelo sistemáticode elección o evaluación de tecnologías, a partir de susescritos y de los aportes de otros defensores deloslímites al crecimiento como Gorz, Latouche, Ullrich y Sachs, es posible trazar las líneas principales de una concepción de la evaluación de tecnologías que sea depositaria de la perspectiva illichiana. Dichas líneas son:

Limitación y pluralismo. Illich consideraque es la misma estructura técnica de las herramientas dominantes propias del modo de producción industrial la que hace imposible su control mediante un proceso político.El predominio de las tecnologías industriales constituye una amenaza para la democracia justamente porque éstas tienden a suprimir las alternativas tecnológicas. Por eso, el espacio de la elección o evaluación de tecnologías solamente se abre tras haber limitado el desarrollo de las herramientas dominantes, tras haber suprimido su monopolio sobre la producción de bienes y servicios, tras haber dejado que prolifere una diversidad de modos de producción autónoma y una diversidad de herramientas convivenciales que les son propias. En este sentido, los defensores de los límites al crecimiento apuestan por un pluralismo radical, según el cual no hay una posibilidad real de elección o evaluación de tecnologías a menos que se abra el libre juego de las alternativas tecnológicas radicalmente diferentes. En este juego pueden participar también las tecnologías industriales, pero ya no les será dado arreglar la jugada de antemano.

Ahora bien, una vez abierto el libre juego de las alternativas tecnológicas y, en consecuencia, el espacio (eminentemente político) de la evaluación de tecnologías radicalmente diferentes, es preciso introducir algunos criterios que pueden contribuir a una justa comparación.

Suficiencia. El primero de estos criterios se basa en la mencionada norma de lo suficiente y, en concordancia con esto, puede designarse como criterio de suficiencia. Este criterio señala las tecnologías que logran un máximo de satisfacción de las necesidades humanas con un mínimo de producción, con un costo social y natural mínimo. Por esta razón, el criterio de suficiencia 
puede considerarse también como un criterio minimalista destinado a establecer la proporcionalidad entre los medios y los fines, esto es, entre los beneficios brindados por las tecnologías y el costo social y natural requerido para su producción, operación y mantenimiento. Esto presupone, por un lado, la abolición del imperativo tecnológico que dicta la realización de cualquier hazaña tecnológica realizable sin importar el costo social y natural que ésta demande y, por el otro, el análisis crítico constante de las necesidades humanas y la determinación del ciclo de producción y consumo en base a las necesidades genuinas, experimentadas, no creadas. La idea de que la producción y el consumo puedan decidirse a partir de las necesidades es, como señala Gorz, una idea "políticamente subversiva", 22 pero esta idea es también filosóficamente subversiva porque pone de manifiesto que la evaluación de tecnologías constituye ante todo una evaluación de necesidades. El criterio de suficiencia trata de establecer así, no sólo una cierta proporcionalidad entre los medios y los fines, sino también una drástica contracción de los fines, habida cuenta de que la mayoría de las necesidades que se ufanan de satisfacer las tecnologías industriales contemporáneas no son más que necesidades creadas con el propósito de acrecentar el ciclo mismo de producción y consumo. Al respecto, Gorz señala que el criterio de suficiencia supone la negación radical de la lógica capitalista porque propugna un sistema económico "fundado en la búsqueda del mínimo derroche posible" 23 o, en otras palabras, fundado en la renuncia, en la mesura, en la sobriedad voluntaria.

Internalización de costos. El segundo de los criterios de evaluación de tecnologías propuesto por Illich y otros defensores de los límites al crecimiento es la internalización de los costos sociales y naturales que demanda la producción, operación y mantenimiento de tecnologías. La expresión "internalización de costos" designa precisamente el requisito de que los costos sociales y naturales de las tecnologías se carguen al aqui y al abora, en lugar de ser externalizados y difusamente esparcidos por una diversidad de espacios y de tiempos, esto es, en lugar de ser transferidos a la naturaleza, a otras sociedades y a las generaciones futuras. De acuerdo con Ullrich, "la capacidad de transferir costos hace posible que la tecnología industrial

22 Gorz, op. cit. (2012), p. 79.

${ }^{23}$ Ibidem, p. 81. 
aparezca en una forma mistificada (...) [y que] cualquier tecnología alternativa, no mistificante, que hace todos sus costos y desventajas inmediatamente palpables al usuario, parezca muy poco atractiva, hasta primitiva." 24

Sin embargo, uno de los propósitos de esta concepción de la evaluación de tecnologías inspirada en la perspectiva filosófica de Illich es justamente detectar los casos de transferencia y ponderar los costos reales de la producción, operación y mantenimiento de tecnologías conjuntando los costos transferidos y no transferidos, los costos externalizados e internalizados. Así, la comparación entre las diferentes alternativas tecnológicas (sean industriales o no industriales) puede comenzar a hacerse en pie de igualdad. De lo anterior se desprenden al menos dos consecuencias: la primera es que la compatibilidad con la naturaleza puede ser unaspecto importante de la internalización de costos, pero no es el único que debe considerarse; la segunda es que, en la medida en que la evaluación de tecnologías tome en cuentalos costos transferidos, es probable queninguna tecnología industrial pueda proclamarsea sí misma como natural y socialmente sostenible.

Autonomía bumana y convivencialidad. El tercero de los criterios de evaluación de tecnologías se basa en la noción illichiana de "convivencialidad". Las tecnologías convivenciales son aquéllas que expanden el radio de acción de los hombres sin degradar la autonomía humana, son aquéllas que no demandan, para su producción, operación y mantenimiento, una estructura que escape al control de las personas y las comunidades. Las tecnologías primitivas en general preservan la autonomía humana pero no son realmente eficientes, mientras que ocurre exactamente lo contrario con las tecnologías propias del modo de producción industrial. De acuerdo con Illich, en este momento histórico estamos en condiciones de concebir tecnologías realmente convivenciales, esto es, tecnologías que estén marcadas al mismo tiempo con el sello de la eficiencia y con el sello del respeto por la autonomía humana. En otras palabras, "podemos concebir herramientas que

${ }^{24}$ Ullrich, Otto, “Tecnología”, en Sachs, op. cit. (1996), pp. 371-372. 
permitan eliminar la esclavitud del hombre frente al hombre, sin someterlo a la máquina." 25

Las tecnologías convivenciales preservan la autonomía humana al menos en los siguientes aspectos:

(1) Energía. Las tecnologías convivenciales utilizan principalmente la energía metabólica humana y la hacen más eficiente; de este modo, evitan que las personas y las comunidades dependan de empresas o instituciones centralizadas que proveen energía externa (no metabólica) como la electricidad, los combustibles derivados del petróleo, etc.

(2) Saber. Las tecnologías convivenciales aprovechan los saberes disponibles de las personas y las comunidades y no las hacen depender significativamente del conocimiento experto en los procesos de producción, operación y mantenimiento de tecnologías; en este sentido, puede decirse que la convivencialidad promueve una mayor dispersión del control técnico y, por eso mismo, se contrapone abiertamente a cualquier forma de tecnocracia.

(3) Permanencia y cambio. Las tecnologías convivenciales son durables y eluden la obsolescencia programada que es característica del modo de producción industrial; sin embargo, también pueden admitir modificaciones y cambios que las hagan compatibles con una diversidad de culturas y de modos de vida. Según Illich, "la renovación de las herramientas convivenciales tendrá la espontaneidad de los seres que las manejen."26

Las tecnologías convivenciales son entonces, por definición, tecnologías que surgen de las interacciones entre los modos industriales y no industriales de producir tecnologías, y que logran unir la eficiencia de las tecnologías industriales con el respeto por la autonomía humana que es propio de las tecnologías no industriales. Como se ha mencionado antes, Illich considera como tecnologías convivenciales a las bicicletas, los libros y los antibióticos, en la medida en que pueden ser incorporados a modos de producción autónoma de la circulación, la educación y la salud. Las innovaciones introducidas en la producción agropecuaria por las

\footnotetext{
${ }^{25}$ Illich, op. cit. (2006), p. 408

${ }^{26}$ Ibidem, p. 447.
} 
comunidades rurales de México,27 los botiquines de medicina herbaria asignados a los pastores nómades de Mongolia, ${ }^{28}$ así como las técnicas de construcción con bloques de tierra comprimida desarrolladasinicialmente en Colombia $^{29}$ y actualmente difundidas por todo el mundo, por citar solamentealgunos ejemplos más, también pueden considerarse como tecnologías convivenciales.

Sin embargo, el aspecto más relevante de esta noción filosóficano reside en su poder descriptivo, es decir, en su poder para identificar casos históricamente documentados de producción de tecnologías convivenciales, sino en su poder normativo, esto es, en su poder para orientar procesos futuros de elección o evaluación de tecnologías y para delinear una agenda de innovación tecnológica que esté marcada con el sello de la convivencialidad.

Recibido: 9/2015; aceptado: 11/2015

27 Barkin, David, Fuente, Mario y Rosas, Mara, "Tradición e innovación. Aportaciones campesinas en la orientación de la innovación tecnológica para forjar sustentabilidad", en Trayectorias, vol. 11, núm. 29, 2009,pp. 39-54.

${ }^{28}$ Organización Mundial de la Salud,Estrategia de la OMS sobre medicina tradicional, OMS, 2013.

${ }^{29}$ Sánchez Gama, Clara, "La arquitectura de tierra en Colombia, procesos y culturas constructivas", en Apuntes, vol. 20, núm. 2, 2007, pp. 242-255. 